\title{
Factor structure, reliability, and validity of the Japanese version of the Hoarding Rating Scale- Self-Report (HRS-SR-J)
}

This article was published in the following Dove Press journal:

Neuropsychiatric Disease and Treatment

9 May 2017

Number of times this article has been viewed

\section{Aki Tsuchiyagaito ${ }^{1-3}$ \\ Satoshi Horiuchi ${ }^{4}$ \\ Toko Igarashi ${ }^{5}$ \\ Yoshiya Kawanori ${ }^{4}$ \\ Yoshiyuki Hirano 1,3 \\ Hirooki Yabe ${ }^{2}$ \\ Akiko Nakagawa ${ }^{1,3}$}

'Research Center for Child Mental Development, Chiba University, Chiba,

${ }^{2}$ Department of Neuropsychiatry, Fukushima Medical University, Fukushima, ${ }^{3}$ United Graduate School of Child Development, Osaka University, Kanazawa University, Hamamatsu University School of Medicine, Chiba University and University of Fukui, Osaka, ${ }^{4}$ Faculty of Social Welfare, Iwate Prefectural University, Iwate, ${ }^{5}$ Graduate School of Education, Joetsu University of Education, Niigata, Japan
Correspondence: Satoshi Horiuchi Faculty of Social Welfare, Iwate Prefectural University, 152-52, Sugo, Takizawa, Iwate 020-0693, Japan Tel +8I 196942300

Email horiuchi@iwate-pu.ac.jp
Background: The Hoarding Rating Scale-Self-Report (HRS-SR) is a five-item scale that assesses the symptoms of hoarding. These symptoms include excessive acquisition, difficulty in discarding, and excessive clutter that causes distress. We conducted three studies to examine the factor structure, reliability, and validity of the Japanese version of the HRS-SR (HRS-SR-J).

Methods: Study 1 examined its reliability; 193 college students and 320 adolescents and adults completed the HRS-SR-J and, of the college students, 32 took it again 2 weeks later. Study 2 aimed to confirm that its scores in a sample of 210 adolescents and adults are independent of social desirability. Study 3 aimed to validate the HRS-SR-J in the aspects of convergent and discriminant validity in a sample of 550 adults.

Results: The HRS-SR-J showed good internal consistency and 2-week test-retest reliability. Based on the nonsignificant correlations between the HRS-SR-J and social desirability, the HRSSR-J was not strongly affected by social desirability. In addition, it also had a good convergent validity with the Japanese version of the Saving Inventory-Revised (SI-R-J) and the hoarding subscale of the Obsessive-Compulsive Inventory, while having a significantly weaker correlation with the five subscales of the Obsessive-Compulsive Inventory, except for the hoarding subscale. In addition, the strength of the correlation between the HRS-SR-J and the Japanese version of the Patient Health Questionnaire-9 and that between the HRS-SR-J and the Generalized Anxiety Disorder-7 were significantly weaker than the correlation between the HRS-SR-J and the SI-R-J. These results demonstrate that the HRS-SR-J has good convergent and discriminant validity.

Conclusion: The HRS-SR-J is a notable self-report scale for examining the severity of hoarding symptoms.

Keywords: Japanese version of the Hoarding Rating Scale-Self-Report, Hoarding Rating Scale, hoarding, reliability, validity

\section{Background}

Hoarding behavior is defined as the excessive acquisition of and the difficulty in discarding items with potentially limited value. ${ }^{1}$ In severe cases, this behavior can lead to cluttering of living spaces, which may cause severe safety, health, financial, housing, and employment issues. In addition, hoarding behavior could induce considerable distress and impair daily functionality, which affects not only the individual exhibiting these behaviors but also their family members and community. ${ }^{2,3}$ Thus, effective management of hoarding behaviors represents an important topic to be addressed within the fields of mental health, public health, and social welfare.

Hoarding is defined as a combination of the following five components: 1) excessive acquisition, 2) difficulty in discarding, 3) clutter of living spaces, 4) impairment 
of daily functionality, and 5) physical, psychological, and social distress. ${ }^{4}$ The prevalence of hoarding among people is now estimated to be between $2 \%$ and $5 \%,,^{2,5,6}$ making it more commonplace than previously thought, with the initial onset of hoarding symptoms being between 11 and 20 years of age..$^{1,7-10}$ The course of hoarding tends to be chronic and progressive, with symptoms often becoming worse over time..$^{8-10}$ To clarify its prevalence and etiology, as well as to ultimately develop effective treatments, reliable and valid assessments must be carried out.

Several self-report measures have been developed to assess the features and severity of hoarding symptoms. For example, the Clutter Image Rating $(\mathrm{CIR})^{11}$ is a visual assessment composed of a series of nine photographs that specifically assess the severity of clutter in the three main rooms (kitchen, living room, and bedroom) in a general home environment. Another example is the Saving InventoryRevised (SI-R), ${ }^{12}$ which consists of three subscales: excessive acquisition, difficulty in discarding, and clutter. These subscales include items that assess impairment and distress, thus assessing all hoarding symptoms. However, the SI-R is somewhat long-winded as a measure for clinical application and research settings.

In contrast, the Hoarding Rating Scale-Self-Report (HRS-SR), ${ }^{13}$ a self-report version of the Hoarding Rating Scale-Interview (HRS-I), ${ }^{14}$ is a briefer measure containing five items, and it specifically assesses the following five symptoms: 1) level of clutter in living spaces, 2) difficulty in discarding possessions, 3 ) excessive acquisition of items, 4) emotional distress regarding hoarding behavior, and 5) significant impairment of functioning, all of which directly align with the Diagnostic and Statistical Manual of Mental Disorders, Fifth Edition (DSM-5) 4 criteria for hoarding disorder. The HRS-SR asks each respondent to rate the severity of their behavior on a 9 -point Likert scale $(0=$ "none/not at all" to $8=$ "extreme") for five features.

The HRS-SR was developed by converting the format of the HRS-I into that of a self-report. ${ }^{13}$ The HRS-I has been found to be a reliable and valid scale. It showed good internal consistency ( $\alpha=0.97)$, test-retest reliability, and cross-context reliability $(r=0.73-0.96) .{ }^{14}$ It also showed strong correlation between scores of the SI-R and the CIR ( $r=0.57-0.94)$, distinguishing participants with and without hoarding symptoms, supporting concurrent and known group validity. ${ }^{14}$ Furthermore, Wootton et $\mathrm{al}^{15}$ examined how the scores of the HRS-I correlated with those of the ObsessiveCompulsive Inventory-Revised (OCI-R). ${ }^{16}$ They found that the scores of the HRS-I correlated strongly with the scores of the items measuring hoarding symptoms ( $r=0.89$ ), while correlating very weakly with those measuring nonhoarding symptoms $(r=-0.01)$.

However, less psychometric information exists (compared to the HRS-I) regarding the HRS-SR. ${ }^{13}$ Its reliability was examined in terms of internal consistency and was found to be high, with a Cronbach's $\alpha$ of 0.83 . A strong correlation $(r=0.92)$ was found between the scores of the HRS-SR and the HRS-I, suggesting good concurrent validity. ${ }^{13}$ However, further studies are needed to clarify the psychometric properties of the HRS-SR. For example, no information is available regarding its factorial validity. The sum of the five items of the HRS-SR is considered as the indicator of the severity of hoarding behavior. ${ }^{13}$ This presupposes that the HRS-SR consists of one factor. In addition, no information exists about its test-retest reliability, and publishing its test-retest data so that users know the level of stability they can rely on is needed. Moreover, convergent and discriminant validity in the HRS-SR has not been examined yet. In addition, the HRS-SR is composed of five items, and it is easy for researchers and clinicians to use it to assess the severity of hoarding behavior. However, there is no existing Japanese language version of the HRS-SR. Therefore, translating the HRS-SR to its Japanese version (HRS-SR-J) could be useful for researchers and clinicians in Japan.

Therefore, the purpose of this study was to examine the reliability and the validity of the HRS-SR-J via factorial validity, test-retest reliability, and convergent and discriminant validity. Thus, this study aims to contribute to the literature of hoarding behavior by adding new information on the psychometric properties of the HRS-SR. Although self-report measures are relatively easy to collect, compared to interview measures, these can be affected by social desirability bias when used for evaluation purposes. ${ }^{17}$ There might also be the potential for bias when asking participants about hoarding behaviors because these behaviors may be perceived as socially undesirable. Therefore, prior to examining the validity of this scale, we checked whether this scale was strongly affected by social desirability. In addition, since hoarding behavior is best conceptualized as a dimensional and continuous construct, which ranges from nonclinical levels to clinically severe hoarding symptoms, ${ }^{18}$ we focused on Japanese nonclinical samples to examine its reliability and validity. We conducted three studies as follows: Study 1 examined the factor structure and reliability of the HRS-SR-J. Study 2 checked whether the HRS-SR-J was affected by social desirability. Study 3 examined the HRS-SR-J's validity. In Study 3, to examine convergent validity, we adopted the 
Japanese version of the SI-R (SI-R-J), ${ }^{12,19}$ which measures the severity of hoarding symptoms; the Japanese version of the CIR (CIR-J), ${ }^{11,20}$ which measures the severity of clutter; and the hoarding subscale of the Japanese version of the OCI (OCI-J). ${ }^{21,22}$ In addition, to examine the discriminant validity, we adopted the other five subscales of the OCI-J for measuring obsessive-compulsive symptoms; the Japanese version of the Patient Health Questionnaire-9 (PHQ-9-J), ${ }^{23,24}$ which measures the severity of depression; and the Japanese version of the Generalized Anxiety Disorder-7 (GAD-7-J), ${ }^{25,26}$ which measures the severity of generalized anxiety disorder.

\section{Ethical considerations}

The research protocol was approved prior to the study by the institutional review board of the Graduate School of Medicine, Chiba University, Japan. All participants gave their written informed consent to participate in this study.

\section{Study I: factor structure and reliability analyses \\ Method}

\section{Translation of the HRS-SR}

The HRS-SR was translated into Japanese via the backtranslation procedure. ${ }^{27}$ To summarize the procedure, first a forward translation from the source language into Japanese was done by authors (TI and AT) fluent in Japanese and English (both of whom majored in Clinical Psychology). Then, a professional English translator, an English/Japanese bilingual who was blind to the original HRS-SR, translated the provisional HRS-SR-J back into English. Then, the two English versions of the HRS-SR (original and back-translated versions) were reconciled by the first translators (TI and AT) and a psychiatrist (AN). Discrepancies between the two versions were low and were discussed until consensus was achieved. One of the original authors (DT) checked the finalized HRS-SR-J (Supplementary materials) and confirmed that the original meaning of each item did not change throughout the translation procedure.

\section{Procedure and participants}

Study 1 was conducted from January to February of 2016. Accordingly, 195 Japanese college students from a northeastern public college participated in a paper-and-pencil survey during classes and privately. Two weeks later, 46 of the 195 students were asked to complete the HRS-SR-J again, and 32 students agreed to participate. To track the same participants, they were asked to fill in the last five digits of their mobile phone number at the time of initial distribution and again asked to fill in that number 2 weeks later. In addition, from the beginning to the middle of March 2016, an online survey was conducted with 320 adolescents and adults. The purposes of the study and the ethical considerations (eg, the right to drop out during the study) were explained to the participants prior to the beginning of the survey. The HRS-SR-J was then distributed among those who agreed to participate.

Of the 195 college students who participated in the paperand-pencil survey, two students were excluded from the analyses due to missing data, and the result of the Web survey was a data set that had no missing data. Therefore, data from 21 male and 172 female college students and that of 210 male and 110 female adolescents and adults from the Web survey were pooled and subjected to analyses of the factor structure and internal consistency reliability. The average age was 20.6 years (with a standard deviation [SD] of 1.76 years for the students) and 34.9 years (with SD of 7.93 years) for the adolescents and adults. The average age of the 32 students who agreed to participate again for establishing the test-retest reliability was 20.8 years, with SD of 1.41 years. There were more females than males in the college sample, and we collected more responses to the Web survey from males than females (total pooled sample was 231 males and 282 females).

\section{Statistical analyses}

Exploratory factor analysis was conducted using IBM SPSS Statistics package 23.0 (SPSS, Inc, Chicago, IL. USA).

\section{Results and discussion \\ Demographic characteristics}

Participants' demographic characteristics are listed in Table 1.

\section{Factor structure}

Exploratory factor analysis with the maximum-likelihood method was conducted. The eigenvalues of the first five factors in the HRS-SR-J were, in descending order, 3.34, $0.63,0.51,0.34$, and 0.18 , accounting for $66.79 \%, 12.62 \%$, $10.24 \%, 9.77 \%$, and $3.59 \%$ of the variances, respectively. This indicated that the HRS-SR-J consisted of one factor. Table 2 shows the factor loading of each item. As shown, all items loaded highly on the factor with values ranging from 0.58 to 0.93 . The results from the factor analysis indicate that the HRS-SR-J is a single-factor scale with five items.

\section{Reliability}

The internal consistency of the HRS-SR-J was evaluated by calculating the Cronbach's $\alpha$, which was found to have a value 
Table I Demographic variables and the total score of the HRS-SR-J

\begin{tabular}{|c|c|c|c|c|c|c|c|c|c|c|c|c|}
\hline \multirow[t]{2}{*}{ Variables } & \multicolumn{4}{|c|}{ Pooled sample } & \multicolumn{4}{|c|}{ College sample } & \multicolumn{4}{|c|}{ Web sample } \\
\hline & $\mathbf{n}$ & $\%$ & Mean & SD & $\mathbf{n}$ & $\%$ & Mean & SD & $\mathbf{n}$ & $\%$ & Mean & SD \\
\hline Total & 513 & 100.00 & 10.55 & 7.86 & 193 & 100.00 & 9.88 & 5.98 & 330 & 100.00 & 10.95 & 8.79 \\
\hline \multicolumn{13}{|l|}{ Gender } \\
\hline Male & 231 & 45.03 & 10.42 & 8.34 & 21 & 10.88 & 9.10 & 5.71 & 210 & 65.63 & 10.55 & 8.56 \\
\hline Female & 282 & 54.97 & 10.65 & 7.47 & 172 & 89.12 & 9.97 & 6.03 & 110 & 34.38 & 11.72 & 9.21 \\
\hline \multicolumn{13}{|c|}{ Age, decades } \\
\hline $10 \mathrm{~s}$ & 89 & 17.35 & 9.78 & 6.20 & 77 & 39.90 & 9.81 & 5.49 & 12 & 3.75 & 9.58 & 9.94 \\
\hline $20 \mathrm{~s}$ & 185 & 36.06 & 10.58 & 7.49 & 115 & 59.59 & 9.81 & 6.22 & 70 & 21.88 & 11.86 & 9.12 \\
\hline $30 \mathrm{~s}$ & 152 & 29.63 & 12.06 & 9.02 & 1 & 0.52 & - & - & $15 \mid$ & 47.19 & 11.99 & 9.00 \\
\hline $40 \mathrm{~s}$ & 87 & 16.96 & 8.62 & 7.56 & 0 & 0.00 & - & - & 87 & 27.19 & 8.62 & 7.59 \\
\hline
\end{tabular}

Abbreviations: HRS-SR-J, Japanese version of the Hoarding Rating Scale-Self-Report; SD, standard deviation.

of 0.87 . Hence, the internal consistency is comparable to the original scale, the alpha of which had a value of 0.83 .

In addition, the correlation coefficients between the scores of the HRS-SR-J at the two time points were calculated to examine the temporal stability of the measure. The coefficient was equal to $0.71(P<0.01)$, indicating that the HRS-SR-J is a reliable scale.

\section{Study 2: social desirability analysis Methods}

\section{Participants and procedures}

In early March 2016, 210 Japanese adolescents and adults were recruited through a Web survey managed by an external company. Explanations regarding the purpose and procedure of the study were given to each participant, as was done in Study 1. Of the participants, $50.0 \%(\mathrm{n}=105)$ were male, and participants had the following age distribution: $9.0 \%(\mathrm{n}=19)$ were in their $10 \mathrm{~s}$ or $20 \mathrm{~s}, 11.4 \%(\mathrm{n}=24)$ in their $30 \mathrm{~s}, 24.8 \%$ $(\mathrm{n}=52)$ in their $40 \mathrm{~s}, 27.6 \%(\mathrm{n}=58)$ in their $50 \mathrm{~s}$, and $27.1 \%$ $(n=57)$ in their 60 s or older. The participants completed both the HRS-SR-J and the simplified version of the Japanese social desirability scale. ${ }^{28,29}$

\section{Measures}

The severity of participants' hoarding behavior was assessed through the HRS-SR-J translated version in Study 1.

Table 2 Summary of exploratory factor analysis

\begin{tabular}{ll}
\hline Items & $\begin{array}{l}\text { Factor } \\
\text { loadings }\end{array}$ \\
\hline I. Clutter & 0.74 \\
2. Difficulty in discarding & 0.70 \\
3. Acquiring & 0.58 \\
4. Distress & 0.84 \\
5. Impairment & 0.93 \\
\% of variance & 66.79 \\
\hline
\end{tabular}

The scores of the five items were summed, with higher scores indicating more severe hoarding behavior.

Social desirability was assessed with a simplified version of the Japanese social desirability scale, ${ }^{28,29}$ which consists of 10 items. An example item was "When I don't know something, I don't at all mind admitting it." Each item was rated "yes" (coded as one) or "no" (coded as zero). The scores of these 10 items were summed, with higher scores again indicating a stronger tendency toward social desirability.

\section{Statistical analyses}

Correlation analyses were conducted using IBM SPSS Statistics package 23.0 (SPSS, Inc). For all tests, a $P$-value of 0.05 (two-tailed) was chosen as the statistically significant cutoff.

\section{Results and discussion}

Correlation analysis was conducted to examine whether the scores would be independent from social desirability, as Redding et $\mathrm{a}^{17}$ suggested that social desirability is one of the participants' attitudes that can cause response bias. The correlation between the HRS-SR-J and social desirability was not significant ( $r=-0.04, P=0.54$ ), and hence, it was found that the scores of the HRS-SR-J were not strongly affected by social desirability.

\section{Study 3: validity analyses Methods}

\section{Procedure and participants}

At the end of March 2016, another online survey was conducted by the same company as Study 2. Explanations regarding the purpose and procedure of the study were given to each participant, as was done in Studies 1 and 2. Accordingly, 550 Japanese adults were recruited; 50\% ( $\mathrm{n}=275)$ participants were male, and the age distribution was as 
follows: $20 \%(n=110)$ were in their $20 \mathrm{~s}, 20 \%(n=110)$ were in their 30 s, $20 \%(n=110)$ were in their 40 s, $20 \%(n=110)$ were in their $50 \mathrm{~s}$, and $20 \%(n=110)$ were in their 60 s. The average age of the participants was 45.1 years, with SD of 13.97 years.

\section{Measures}

In addition to the HRS-SR-J, the following scales were measured.

1. The Japanese version of the SI-R (SI-R-J) ${ }^{12,19}$

The SI-R-J ${ }^{22}$ is a 23 -item self-report scale utilizing the five-point Likert scale from zero to four $(0=$ "no presence of hoarding behavior or symptoms" to $4=$ "extreme hoarding behavior or symptoms"). Factor analysis has revealed three subscales: excessive acquisition, difficulty in discarding, and clutter. Its reliability and validity have been established. ${ }^{19}$ In this study, we used the SI-R-J alongside the HRS-SR-J to assess convergent validity. A strong positive correlation between each subscale of the SI-R-J and each subscale of the HRS-SR-J was expected. We sought to examine the relationships among the three corresponding HRS-SR-J and SI-R-J subscales: clutter, difficulty in discarding, and acquisition. We calculated partial correlations among pairs of subscales, while controlling for the other two SI-R subscales. In line with a previous study, ${ }^{14}$ we predicted that the clutter subscale of the HRS-SR-J correlated with the clutter and difficulty in discarding subscales of the SI-R-J, that the difficulty in discarding subscale of the HRS-SR-J correlates significantly with the clutter and difficulty in discarding of the SI-R-J, and that the acquisition subscale of the HRS-SR-J correlates significantly with the acquisition subscale of the SI-R-J.

2. The Japanese version of the CIR (CIR-J) ${ }^{11,20}$

The CIR-J ${ }^{20}$ consists of nine photographs of increasing clutter severity for the three living spaces: kitchen, living room, and bedroom, similar to the original CIR, ${ }^{11}$ and has adequate reliability and validity. ${ }^{20}$ Clutter severity was then assessed by asking participants to choose one of the nine photographs that most closely resembles the level of clutter in their three rooms. The three scores were then averaged to make a total score for each person. In this study, we used the CIR-J to check the convergent validity. We expected to find a strong positive correlation between the CIR-J and the HRS-SR-J.

3. The Japanese version of the OCI (OCI-J) $)^{21,22}$

The OCI-J ${ }^{22}$ is a 42 -item self-report scale used to assess the severity of a participant's obsessive-compulsive symptoms. The OCI-J has shown evidence of reliability and validity. ${ }^{22}$ Each of its items is rated on a five-point Likert scale corresponding to the frequency of symptoms in the past month and the severity of distress $(0=$ "not at all distressed" to 4= "extremely distressed"). Factor analysis has revealed six subscales: checking, doubting, ordering, obsessing, neutralizing, and hoarding. In this study, based on the findings of a previous study, ${ }^{15}$ we expected to find a strong positive correlation between the hoarding subscale of the OCI (OCI-HD-J) and the HRS-SR-J, indicating convergent validity. In addition, the other five subscales of the OCI (OCI-OCD-J) and the HRS-SR-J were expected to exhibit a significantly weaker positive correlation than the correlation between the OCI-HD-J and the HRS-SR-J, indicating the discriminant validity.

4. The Japanese version of the Patient Health Questionnaire-9 (PHQ-9-J) $)^{23,24}$

The PHQ-9- $\mathrm{J}^{27}$ consists of nine items assessing the severity of depression. The PHQ-9-J has shown evidence of reliability and validity. ${ }^{24}$ Each item is rated on a fivepoint Likert-type scale corresponding to the frequency of symptoms in the past 2 weeks $(0=$ "not at all" to $4=$ "nearly every day"). In this study, we used the PHQ-9-J to check the discriminant validity. We expected to find the correlation between PHQ-9-J and the HRS-SR-J to be significantly weaker than the correlation between the SI-R-J and the HRS-SR-J.

5. The Japanese version of the Generalized Anxiety Disorder-7 (GAD-7-J) $)^{25,26}$

The GAD-7- $\mathrm{J}^{29}$ consists of seven items that measure the severity of generalized anxiety disorder. The GAD-7-J has shown evidence of reliability and validity. ${ }^{26}$ Each item is rated on a four-point Likert-type scale corresponding to the frequency of symptoms in the past 2 weeks $(0=$ "not at all" to $4=$ "nearly every day"). In this study, the GAD-7-J was used to check discriminant validity. We expected to find the correlation between the GAD-7-J and the HRS-SR-J to be significantly weaker than the correlation between the SI-R-J and the HRS-SR-J.

\section{Statistical analyses}

Correlation analyses were conducted using IBM SPSS Statistics package 23.0 (SPSS, Inc). For all tests, a $P$-value of 0.05 (two-tailed) was chosen as the statistically significant cutoff. In addition, Cohen's criteria were applied to judge the Pearson correlation coefficients (weak correlation: $|r| \geq 0.10$, moderate correlation: $|r| \geq 0.30$, and strong correlation: $|r| \geq 0.50) .^{30}$ 


\section{Results and discussion}

\section{Convergent validity}

Table 3 shows the Pearson correlation coefficients of the HRS-SR-J with the SI-R-J, the CIR-J, the OCI-HD-J, the OCI-OCD-J, the PHQ-9-J, and the GAD-7-J scales. The HRS-SR-J scales had significantly strong positive correlations with the SI-R-J scales ( $r=0.54-0.84)$. However, contrary to our expectations, the correlation between the HRS-SR-J and the CIR-J was only moderately positive ( $r=0.33-0.41)$. Table 4 shows the partial correlations among pairs of subscales of the three corresponding HRS-SR-J and SI-R-J subscales, controlling for the other two SI-R subscales. Contrary to our expectations, the clutter subscale of the HRS-SR-J correlated with the acquisition subscale, as well as clutter and difficulty in discarding subscales of the SI-R-J. Other than this unexpected correlation, the partial correlations among the three corresponding HRS-SR-J and SI-R-J subscales were mostly consistent with our hypothesis. All three HRS-SR-J subscales showed the strongest correlations with their corresponding SI-R-J subscales. Moreover, as shown in Table 3, the HRS-SR-J had a moderate-to-strong positive correlation with the OCI-HD-J ( $r=0.47-0.59)$, indicating good convergent validity.

\section{Discriminant validity}

As shown in Table 3, the HRS-SR-J had a weaker correlation with the OCI-OCD-J ( $r=0.36-0.49)$, compared to the correlation with the OCI-HD-J. Using Fisher's $r$-to- $z$ transformations to examine differences between pairs of correlations, we found that the correlations between the HRS-SR-J and the OCI-OCD-J were significantly lower than the correlations between the HRS-SR-J and the OCI-HD-J, with $z=2.42-2.92$, $P<0.05$, except for the clutter subscale and the impairment subscale of the HRS-SR-J (with $z=1.82$ and 1.36, not significant). In addition, to examine the relationships between the HRS-SR-J and the OCI-HD-J, we calculated the partial correlations controlling for the OCI-OCD-J. To examine the relationships between the HRS-SR-J and the OCI-OCD-J, we calculated partial correlations, controlling for the OCI-HD-J (Table 5). Using Fisher's $r$-to- $z$ transformations to examine differences between pairs of correlations, we found that correlations between the HRS-SR-J and the OCI-OCD-J $(r=-0.05$ to 0.12 ) were significantly weaker than the correlations between the HRS-SR-J and the OCI-HD-J ( $r=0.29-0.39)$, with $z=3.31-7.64, P<0.01$. Furthermore, the positive correlation between the HRS-SR-J and the PHQ-9-J was weak to moderate ( $r=0.28-0.37$ ), and the correlations were very similar to those between the HRS-SR-J and the GAD-7-J $(r=0.27-0.34)$ (Table 3). Using Fisher's $r$-to- $z$ transformations to examine differences between pairs of correlations, we found that both groups of correlations were significantly weaker than the correlations between the SI-R-J and the HRSSR-J ( $r=0.54-0.84)$, with $z=8.65-13.79, P<0.01$.

\section{General discussion}

The purpose of this study was to examine the factor structure, reliability, and validity of the HRS-SR-J. This study set out to increase the available psychometric information

Table 3 Correlations between the HRS-SR-J and other measures

\begin{tabular}{|c|c|c|c|c|c|c|c|c|c|c|c|c|c|c|c|c|c|c|}
\hline Items & Scale & Mean & (SD) & $\mathbf{I}$ & 2 & 3 & 4 & 5 & 6 & 7 & 8 & 9 & 10 & II & 12 & 13 & 14 & 15 \\
\hline I & HRS-SR-J_Total & 9.99 & (8.98) & - & & & & & & & & & & & & & & \\
\hline 2 & HRS-SR-J_Clutter & 2.00 & $(2.18)$ & 0.89 & - & & & & & & & & & & & & & \\
\hline 3 & HRS-SR-J_Difficulty discarding & 2.03 & $(2.13)$ & 0.86 & 0.81 & - & & & & & & & & & & & & \\
\hline 4 & HRS-SR-J_Acquisition & 2.14 & $(1.96)$ & 0.79 & 0.57 & 0.59 & - & & & & & & & & & & & \\
\hline 5 & HRS-SR-J_Distress & 2.04 & $(2.11)$ & 0.90 & 0.72 & 0.66 & 0.64 & - & & & & & & & & & & \\
\hline 6 & HRS-SR-J_Impairment & 1.79 & $(1.96)$ & 0.91 & 0.74 & 0.66 & 0.65 & 0.89 & - & & & & & & & & & \\
\hline 7 & SI-R-J_Total & 26.60 & $(16.99)$ & 0.83 & 0.72 & 0.67 & 0.69 & 0.77 & 0.77 & - & & & & & & & & \\
\hline 8 & SI-R-J_Acquisition & 8.01 & $(4.98)$ & 0.70 & 0.58 & 0.54 & 0.62 & 0.63 & 0.66 & 0.91 & - & & & & & & & \\
\hline 9 & SI-R-J_Difficuty discarding & 9.10 & $(5.42)$ & 0.74 & 0.61 & 0.65 & 0.64 & 0.67 & 0.65 & 0.92 & 0.81 & - & & & & & & \\
\hline 10 & SI-R-J_Clutter & 9.50 & (7.91) & 0.84 & 0.76 & 0.66 & 0.64 & 0.80 & 0.80 & 0.94 & 0.78 & 0.79 & - & & & & & \\
\hline II & CIR-J_Mean & 1.75 & $(1.07)$ & 0.41 & 0.39 & 0.33 & 0.34 & 0.35 & 0.35 & 0.43 & 0.35 & 0.37 & 0.45 & - & & & & \\
\hline 12 & OCl-HD-J & 2.36 & $(2.45)$ & 0.59 & 0.47 & 0.47 & 0.52 & 0.55 & 0.55 & 0.76 & 0.72 & 0.73 & 0.68 & 0.34 & - & & & \\
\hline 13 & OCl-OCD-J & 24.99 & $(28.94)$ & 0.47 & 0.38 & 0.36 & 0.38 & 0.44 & 0.49 & 0.61 & 0.62 & 0.52 & 0.56 & 0.28 & 0.78 & - & & \\
\hline 14 & PHQ-9-J_Total & 4.84 & $(5.73)$ & 0.36 & 0.30 & 0.28 & 0.29 & 0.34 & 0.37 & 0.46 & 0.45 & 0.28 & 0.44 & 0.27 & 0.55 & 0.72 & - & \\
\hline 15 & GAD-7-J_Total & 3.31 & $(4.5 I)$ & 0.34 & 0.28 & 0.27 & 0.29 & 0.31 & 0.32 & 0.42 & $0.4 I$ & 0.37 & 0.38 & 0.23 & 0.52 & 0.67 & 0.79 & - \\
\hline
\end{tabular}

Note: All $P<0.01$.

Abbreviations: HRS-SR-J, Japanese version of the Hoarding Rating Scale-Self-Report; SI-R-J, Japanese version of the Saving Inventory-Revised; CIR-J, Japanese version of the Clutter Image Rating; OCl-HD-J, hoarding subscale of the Japanese version of the Obsessive-Compulsive Inventory; OCl-OCD-J, five subscales of the Japanese version of the Obsessive-Compulsive Inventory, except for hoarding subscale; PHQ-9-J, Japanese version of the Patient Health Questionnaire-9; GAD-7-J, Japanese version of the Generalized Anxiety Disorder-7. 
Table 4 Correlations between the three subscales of the HRS-SR-J with the corresponding subscales of the SI-R-J, controlling for the other SI-R-J subscales

\begin{tabular}{llll}
\hline Scale & $\begin{array}{l}\text { SI-R-J_Clutter (controlling } \\
\text { for difficulty discarding } \\
\text { and acquisition) }\end{array}$ & $\begin{array}{l}\text { SI-R-J_Difficulty } \\
\text { discarding (controlling for } \\
\text { clutter and acquisition) }\end{array}$ & $\begin{array}{l}\text { SI-R-J_Acquisition } \\
\text { (controlling for clutter } \\
\text { and difficulty discarding) }\end{array}$ \\
\hline HRS-SR-J_Clutter & $0.54^{*}$ & 0.04 & -0.03 \\
HRS-SR-J_Difficulty discarding & $0.33^{*}$ & $0.28^{*}$ & -0.09 \\
HRS-SR-J_Acquisition & $0.22^{*}$ & $0.19 *$ & $0.14^{*}$ \\
\hline
\end{tabular}

Note: $* p<0.01$.

Abbreviations: HRS-SR-J, Japanese version of the Hoarding Rating Scale-Self-Report; SI-R-J, Japanese version of the Saving Inventory-Revised.

on the HRS-SR, a potentially useful self-report measure of hoarding behavior. The factor structure of the HRS-SR-J was examined using exploratory factor analysis and found to be composed of one factor, labeled as hoarding behavior (as expected). Previous studies have used both the total scores of the five items and each score independently. ${ }^{10,13}$ However, demonstration of the scale's unidimensionality provides a rationale for researchers and practitioners to sum up (and average) the scores of the five items.

Reliability was examined in terms of internal consistency and test-retest reliability. The Cronbach's $\alpha$ value of 0.83 was comparable to that of the original HRS-SR, and testretest reliability was high $(r=0.71)$. In addition, the total score of the HRS-SR-J was relatively stable. It was found to be possible to use the HRS-SR-J for understanding and/or assessing the changes of hoarding severity over time. These results indicate that the HRS-SR-J is a reliable measure for assessing hoarding behavior in Japan.

Validity was examined in terms of convergent and discriminant validity. In agreement with our expectations, the HRS-SR-J had a strong positive correlation with the SI-R-J. However, concerning the convergent validity, the correlation between the HRS-SR-J and the CIR-J was weaker than in the previous study. ${ }^{14}$ In our study, the participants were nonclinical, most of them did not have serious hoarding symptoms, and the scores of CIR-J were low, which may explain the relatively weak correlation between the HRS-SR-J and the CIR-J. In addition, the partial correlations among pairs of subscales of the three corresponding HRS-SR-J and SI-R-J subscales, controlling for the other two SI-R-J subscales, were mostly consistent with those of a previous study. ${ }^{14}$ All three HRS-SR-J subscales showed the strongest correlations with their corresponding SI-R-J subscales. However, contrary to our expectations, the clutter subscale of the HRS-SR-J correlated with all three subscales of the SI-R-J. Again, in our study, the participants were nonclinical, and their average scores on the subscales of the HRS-SR-J were not high, relative to hoarding patients. Therefore, we could not find distinguishing characteristics regarding clutter severity, and the clutter subscale of the HRS-SR-J significantly correlated with all three subscales of the SI-R-J.

In terms of the discriminant validity, after controlling for the OCI-OCD-J, the HRS-SR-J had a moderate-to-strong positive correlation with the OCI-HD-J, while having a significantly weaker correlation with the OCI-OCD-J, after controlling for the OCI-HD-J. Moreover, the strength of the correlation between the HRS-SR-J and the PHQ-9-J and that between the HRS-SR-J and the GAD-7-J were significantly weaker than that between the HRS-SR-J and the SI-R-J. Thus, the current findings show that the HRS-SR-J is a valid scale.

\section{Limitations}

One potential limitation of this study was that the participants were recruited from a nonclinical population. Although the

Table 5 Correlations between the HRS-SR-J with the OCl-HD-J and the OCl-OCD-J, controlling for other measures

\begin{tabular}{lll}
\hline Scale & OCI-HD-J (controlling for OCI-OCD-J) & OCI-OCD-J (controlling for OCI-HD-J) \\
\hline HRS-SR-J_Clutter & $0.29 *$ & 0.04 \\
HRS-SR-J_Difficulty discarding & $0.33^{*}$ & -0.01 \\
HRS-SR-J_Acquisition & $0.39^{*}$ & -0.05 \\
HRS-SR-J_Distress & $0.36^{*}$ & 0.03 \\
HRS-SR-J_Impairment & $0.31^{*}$ & $0.12^{*}$ \\
\hline
\end{tabular}

Note: $* p<0.01$.

Abbreviations: HRS-SR-J, Japanese version of the Hoarding Rating Scale-Self-Report; OCI-HD-J, Hoarding subscale of the Japanese version of the Obsessive-Compulsive Inventory; OCl-OCD-J, five subscales of the Japanese version of the Obsessive-Compulsive Inventory, except for hoarding subscale. 
psychometric properties of the HRS-SR-J were promising, the tool did not indicate whether the results could be generalized to a clinical population. Given that the HRS-SR-J would be used in the clinical assessment and treatment of hoarding disorder, it is necessary to assess the psychometric properties of the HRS-SR-J and to replicate the examination of factor structure, reliability, and validity using a clinical population. Despite these limitations, our results are valuable as this is the first study to offer a reliable and valid assessment tool to briefly understand the severity and risk associated with hoarding behavior in Japan.

\section{Acknowledgments}

This work was supported by grants from Japan Society for the Promotion of Science (JSPS) KAKENHI (through grant number JP16H07098) and the Initiative for Realizing Diversity in the Research Environment to the first author, as well as by a faculty research grant from the Iwate Prefectural University (Gakubu-tou-kenkyuhi) to the second author.

\section{Disclosure}

The authors report no conflicts of interest in this work.

\section{References}

1. Frost RO, Gross RC. The hoarding of possessions. Behav Res Ther. 1993;31(4):367-381.

2. Samuels JF, Bienvenu OJ, Grados MA, et al. Prevalence and correlates of hoarding behavior in a community-based sample. Behav Res Ther. 2008;46(7):836-844.

3. Tolin DF, Meunier SA, Frost RO, Steketee G. Hoarding among patients seeking treatment for anxiety disorders. J Anxiety Disord. 2011;25(1):43-48.

4. American Psychiatric Association. Diagnostic and Statistical Manual of Mental Disorders (DSM-5). Washington DC: American Psychiatric Association; 2013.

5. Iervolino AC, Perroud N, Fullana MA, et al. Prevalence and heritability of compulsive hoarding: a twin study. Am J Psychiatry. 2009; 166(10):1156-1161.

6. Mueller A, Mitchell JE, Crosby RD, Glaesmer H, de Zwaan M. The prevalence of compulsive hoarding and its association with compulsive buying in a German population-based sample. Behav Res Ther. 2009;47(8):705-709.

7. Samuels J, Joseph Bienvenu OIII, Riddle MA, et al. Hoarding in obsessive compulsive disorder: results from a case-control study. Behav Res Ther. 2002;40(5):517-528.

8. Grisham JR, Frost RO, Steketee G, Kim HJ, Hood S. Age of onset of compulsive hoarding. J Anxiety Disord. 2006;20(5):675-686.

9. Ayers CR, Saxena S, Golshan S, Wetherell JL. Age at onset and clinical features of late life compulsive hoarding. Int J Geriatr Psychiatry. 2010; 25(2):142-149.

10. Tolin DF, Meunier SA, Frost RO, Steketee G. Course of compulsive hoarding and its relationship to life events. Depress Anxiety. 2010;27(9):829-838.
11. Frost RO, Steketee G, Tolin DF, Renaud S. Development and validation of the clutter image rating. J Psychopathol Behav Assess. 2008; 30(3):193-203.

12. Frost RO, Steketee G, Grisham J. Measurement of compulsive hoarding: saving inventory-revised. Behav Res Ther. 2004;42(10):1163-1182.

13. Tolin DF, Frost RO, Steketee G, Fitch KE. Family burden of compulsive hoarding: results of an internet survey. Behav Res Ther. 2008; 46(3):334-344.

14. Tolin DF, Frost RO, Steketee G. A brief interview for assessing compulsive hoarding: the Hoarding Rating Scale-Interview. Psychiatry Res. 2010;178(1):147-152.

15. Wootton BM, Diefenbach GJ, Bragdon LB, Steketee G, Frost RO, Tolin DF. A contemporary psychometric evaluation of the Obsessive Compulsive Inventory-Revised (OCI-R). Psychol Assess. 2015;27(3): 874-882.

16. Foa EB, Huppert JD, Leiberg S, et al. The Obsessive-Compulsive Inventory: development and validation of a short version. Psychol Assess. 2002;14(4):485-496.

17. Redding CA, Maddock JE, Rossi JS. The sequential approach to measurement of health behavior constructs: issues in selecting and developing measures. Californian J Health Promot. 2006;4(1):83-101.

18. Timpano KR, Broman-Fulks JJ, Glaesmer H, et al. A taxometric exploration of the latent structure of hoarding. Psychol Assess. 2013; 25(1):194-203.

19. Tsuchiyagaito A, Kuromiya K, Igarashi T, et al. A consideration of clinical characteristics of non-clinical hoarding among Japanese adolescents [Japanese]. Anxiety Disord Res. 2015;6(2):72-85.

20. Tsuchiyagaito A, Shimizu E, Nakagawa A. Psychometric properties of the Clutter Image Rating among Japanese adolescents. The 22nd Annual Conference for the International Obsessive-Compulsive Foundation. Boston, MA: Westin Boston Waterfront; 2015.

21. Foa EB, Kozak MJ, Salkovskis PM, Coles ME, Amir N. The validation of a new obsessive-compulsive disorder scale: the ObsessiveCompulsive Inventory. Psychol Assess. 1998;10(3):206-214.

22. Ishikawa R, Kobori O, Shimizu E. Development and validation of the Japanese version of the obsessive-compulsive inventory. BMC Res Notes. 2014;7(1):306.

23. Kessler RC, Andrews G, Colpe LJ, et al. Short screening scales to monitor population prevalences and trends in non-specific psychological distress. Psychol Med. 2002;32(6):959-976.

24. Muramatsu K, Miyaoka H, Kamijima K, et al. The patient health questionnaire, Japanese version: validity according to the mini-international neuropsychiatric interview-plus. Psychol Rep. 2007;101(3 pt 1): 952-960.

25. Spitzer RL, Kroenke K, Williams JBW, Löwe B. A brief measure for assessing generalized anxiety disorder. Arch Intern Med. 2006; 166(10):1092.

26. Muramatsu K, Miyaoka H, Ueshima K, et al. Validation and utility of a Japanese version of the GAD - 7. Jpn J Psychosom Med. 2010; 50(6): 166

27. Storheim K, Brox JI, Løchting I, Werner EL, Grotle M. Crosscultural adaptation and validation of the Norwegian version of the Core Outcome Measures Index for low back pain. Eur Spine J. 2012; 21(12):2539-2549.

28. Crowne DP, Marlowe D. A new scale of social desirability independent of psychopathology. J Consult Psychol. 1960;24(4):349-354.

29. Kitamura T, Suzuki T. Japanese version of Social Desirability Scale. Jpn J Soc Psychiatry. 1986;9:173-180.

30. Cohen J. Statistical power analysis for the behavioral sciences. Stat Power Anal Behav Sci. 1988;2:567. 
Neuropsychiatric Disease and Treatment

Dovepress

\section{Publish your work in this journal}

Neuropsychiatric Disease and Treatment is an international, peerreviewed journal of clinical therapeutics and pharmacology focusing on concise rapid reporting of clinical or pre-clinical studies on a range of neuropsychiatric and neurological disorders. This journal is indexed on PubMed Central, the 'PsycINFO' database and CAS,

and is the official journal of The International Neuropsychiatric Association (INA). The manuscript management system is completely online and includes a very quick and fair peer-review system, which is all easy to use. Visit http://www.dovepress.com/testimonials.php to read real quotes from published authors.

Submit your manuscript here: http://www.dovepress.com/neuropsychiatric-disease-and-treatment-journal 